ISSN 1996-1073

www.mdpi.com/journal/energies

Article

\title{
Optimization of a Fuzzy-Logic-Control-Based Five-Stage Battery Charger Using a Fuzzy-Based Taguchi Method
}

\author{
Chun-Liang Liu ${ }^{1}$, Yi-Shun Chiu ${ }^{1}$, Yi-Hua Liu ${ }^{1, *}$, Yeh-Hsiang $\mathrm{Ho}^{2}$ and Shu-Syuan Huang ${ }^{2}$ \\ 1 Department of Electrical Engineering, National Taiwan University of Science and Technology, \\ EE-105-1 \#No.43, Sec. 4, Keelung Rd., Da’an Dist., Taipei City 10600, Taiwan; \\ E-Mails: d10007201@mail.ntust.edu.tw (C.-L.L.); d10107203@mail.ntust.edu.tw (Y.-S.C.) \\ 2 Electric Energy Technology Division Power Electronics Department, Industrial Technology \\ Research Institute, Rm\#841, Bldg. 51, No. 195, Sec. 4, Chung Hsing Rd., Chutung, Hsinchu 31040, \\ Taiwan; E-Mails: hoyehhsiang@itri.org.tw (Y.-H.H.); elsahuang@itri.org.tw (S.-S.H.) \\ * Author to whom correspondence should be addressed; E-Mail: yhliu@mail.ntust.edu.tw; \\ Tel.: +886-2-27333141 (ext. 1252); Fax: +886-2-27376699.
}

Received: 6 June 2013; in revised form: 9 July 2013 / Accepted: 9 July 2013 /

Published: 17 July 2013

\begin{abstract}
Lithium ion (Li-ion) batteries have been widely used in various kinds of applications, including consumer electronics, green energy systems and electrical vehicles. Since the charging method has a significant influence on the performance and lifetime of Li-ion batteries, an intelligent charging algorithm which can properly determine the charging current is essential. In this study, a fuzzy-logic-control-based (FLC-based) five-stage Li-ion battery charger is proposed. The proposed charger takes the temperature rise and the gradient of temperature rise of battery into account, and adjusts the charging current accordingly. To further improve the performance of the proposed FLC, the fuzzy-based Taguchi method is utilized to determine the optimal output membership functions (MFs). Comparing with the conventional constant current-constant voltage (CC-CV) method, the charging time, charging efficiency, average temperature rise and the obtained cycle life of the Li-ion battery are improved by about $58.3 \%, 1.65 \%, 26.7 \%$ and $59.3 \%$, respectively.
\end{abstract}

Keywords: fuzzy logic control; five-stage constant current charging algorithm; Taguchi method 


\section{Introduction}

Secondary batteries are commonly utilized as energy storage solutions for modern technologies such as portable devices, renewable energy systems and electric vehicles. Among secondary batteries, the lithium ion (Li-ion) battery has become most popular because it offers many advantages such as high power and energy density, high open circuit voltage, low self-discharge rate, low maintenance requirement and no memory effect. Recent trends show that Li-ion batteries are not only widely used in portable consumer electronics, but are also gaining popularity for renewable energy, electric vehicle and military applications. According to the literature, charging methods have a significant influence on the performance and lifetime of Li-ion batteries. Therefore, designing a high-quality battery charger is essential. The objectives of a high-quality charger include high charging efficiency, short charging time and prolonged cycle life [1-5].

The commonly adopted charging method for Li-ion batteries is the constant current-constant voltage (CC-CV) method. For the CC-CV method, a large constant current is applied at the beginning of the charging cycle when the battery state of charge (SOC) is low. When the battery voltage raises to some predefined limit, the charger switches to constant voltage charging mode and continues in that mode until the charging current decreases to a predetermined small value. Although the $\mathrm{CC}-\mathrm{CV}$ is simple to implement, it is not suitable for rapid charging because CV charging seriously extends the overall charging time and also reduces the cycle life of the battery.

To deal with this problem, many advanced battery charging techniques are proposed in the literature [6-20]. In [6] and [7], PLL-based battery chargers (PLBC) are proposed. Based on PLBC, optimal pulse frequency and optimal duty cycle can then be determined using sweeping method to improve the performance of the PLBC [8,9]. In addition, the effect of the internal resistance on the open circuit voltage is also taken into account in [10] to improve the charging speed of PLBC. In [11] and [12], a sinusoidal-ripple current charging strategy is proposed; this approach can minimize the battery impedance and consequently maximize the energy transfer efficiency. The reason for using pulsed charging and sinusoidal current charging technique is that the internal pressures obtained by pulsed charging and sinusoidal current charging are smaller than that obtained by CC charging [6-12]. However, these methods require dedicated hardware to generate pulsed or sinusoidal charging current. In addition, these methods are not truly "adaptive" because additional "search mode" should be utilized to determine the optimal pulse frequency/duty cycle value [8,9]. Another way which can be utilized to reduce the charging time is to adjust the charging current in accordance with the battery status using an intelligent controller. To achieve this goal, an fuzzy-based controller is employed in [13] and a grey-predicted controller is applied in [14]. In both [13] and [14], the proposed intelligent controllers only replace the $\mathrm{CV}$ mode in $\mathrm{CC}-\mathrm{CV}$ charging method, thus the charging current in $\mathrm{CC}$ mode is kept constant. In [15], a genetic algorithm trained generalized regression neural network is used to determine the charging current of an ultra-fast charger. The proposed charger can significantly reduce the charging time; however, it can only be applied to Ni-Cd battery. Global optimization techniques, including ant colony algorithm and Taguchi approach, are proposed to obtain the optimal multi-stage CC charging profile [16-18]. These methods intend to find an optimal charging pattern covering the whole charging process and do not regulate charging current in real time. In [19], a new digital-controlled technique for battery charger is presented to achieve CC-CV control without 
requiring current feedback. In [20], a DSP-based probabilistic fuzzy neural network controller is proposed to replace the traditional proportional-integral controller. The transient of voltage regulation during load variation can be significantly improved.

From the descriptions above, it is obvious that an intelligent charging algorithm which can properly determine the charging current according to measured battery parameters is essential. In this study, a fuzzy-logic-control-based (FLC-based) five-stage Li-ion battery charger is proposed based on the concept presented in [21]. The proposed charger takes the temperature rise and the gradient of temperature rise of battery into account, and adjusts the charging current accordingly. Recently, fuzzy logic controllers (FLCs) have been successfully applied to various fields of engineering. FLCs are robust and are relatively simple to design as they do not require the knowledge of the accurate mathematical model [22-26]. The proposed FLC-based controller is then improved by using Taguchi method to optimize the output membership function (MF) of the fuzzy system. The Taguchi method provides a systematic and effective way for conducting experiments to determine optimal values [17,27]. By using orthogonal arrays (OAs), the Taguchi method can search in the parameter space with a reduced number of experiments [18,28]. Since the aim of this study is to develop an intelligent charger with short charging time, high charging efficiency and low temperature increment; therefore, the optimization can be regarded as a multiple performance characteristic index (MPCI) problem [29,30]. To solve this kind of problem, fuzzy logic is combined with Taguchi method to obtain the optimal output MF of the proposed FLC-based battery charger. The design procedures will be described in detail, and experiments will be carried out to validate the effectiveness of the proposed methods. According to the experimental results, the proposed FLC-based battery charger is capable of charging Li-ion batteries to $85 \%$ capacity in 43.6 min. Comparing to conventional $\mathrm{CC}-\mathrm{CV}$ and multi-stage $\mathrm{CC}$ charging strategy, the charging time, charging efficiency, maximum temperature increment and the lifetime of the Li-ion battery can be improved.

\section{System Configuration}

Figure 1 shows the block diagram of the proposed battery charger. In Figure 1, the input power source of the proposed Li-ion battery charger is a commercially available adaptor, and the battery used is a $4.2 \mathrm{~V}, 2000 \mathrm{mAh} 18650 \mathrm{Li}$-ion battery for notebook computers. From Figure 1, the whole system can be divided into three major parts: (a) input/output interfacing unit; (b) digital control unit and (c) power conversion unit. Detailed descriptions about each unit will be given in the following subsections:

(a) Input/output interfacing unit: the I/O interfacing unit includes feedback circuits which are used to measure the voltage, current and temperature of the battery, and signal conditioning circuits which perform amplification and range adaptation on feedback signals. In this study, the battery voltage and current are sensed using differential amplifier circuits. The temperature is sensed using LM35 precision temperature sensor IC from Texas Instrument Corp. In order to record the long-term experimental data, a graphical user interface (GUI) based on LabVIEW from National Instruments Corp. is also developed to offer a user-friendly monitoring interface of the proposed charger.

(b) Digital control unit: the digital control unit regulates the charging current according to the battery status. In this study, the dsPIC33FJ16GS502 digital signal controller (DSC) from Microchip 
Corp. is used to implement the proposed charging algorithm. PWM modulation strategies and interfacing IC driving signals are also realized using the same DSC to achieve better performance. After the required battery status data (voltage, current and temperature) is acquired, proper charging current can then be determined through the proposed FLC-based battery charger. Detailed description of the software part will be provided in Section 3.

(c) Power conversion unit: a power conversion unit is used to supply the electric power to the Li-ion battery. As shown in Figure 1, a simple buck type DC-DC converter is used as the charging unit. By adequately controlling the PWM gating signal, the power conversion unit can transfer the required energy to the Li-ion battery. The design and implementation of this part of circuit is conventional; therefore, it will not be discussed further here.

Figure 1. Hardware configuration of the proposed charger.

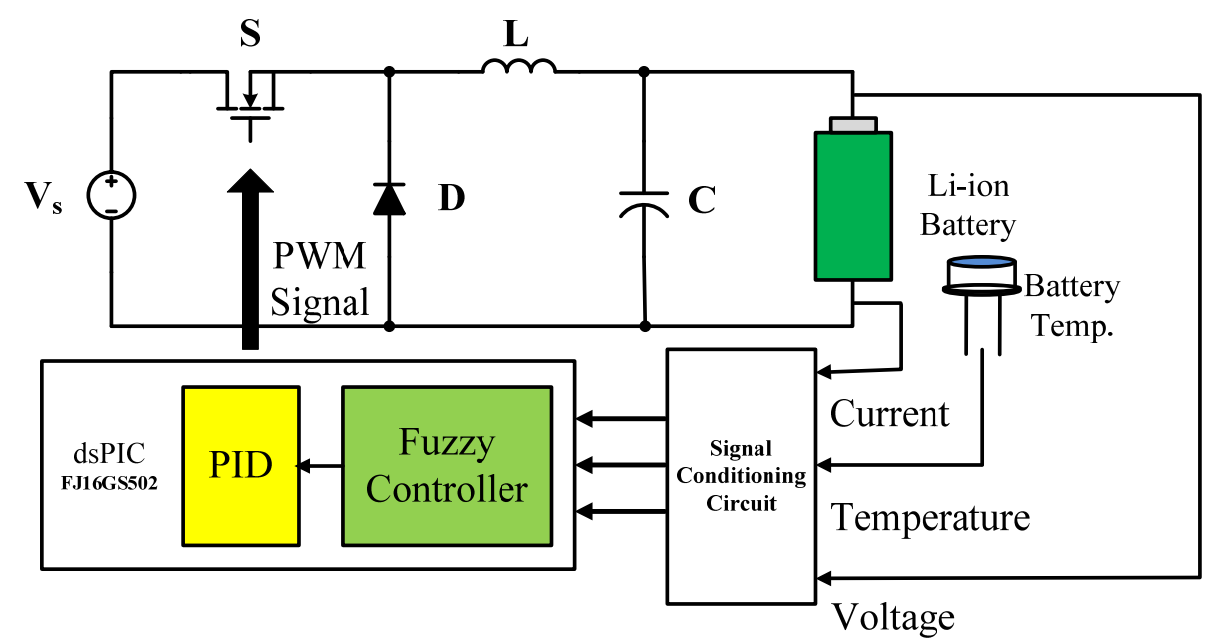

\section{FLC-Based Five-Stage Li-Ion Battery Charger}

According to the literature, the multi-stage $\mathrm{CC}$ charging algorithm has certain advantages such as long cycle life, high charging efficiency and short charging time. However, due to the complex electrochemical characteristics of the Li-ion batteries, the optimal charging current for each stage is hard to obtain [16-18]. The Taguchi method is utilized by the authors in [17] to obtain an optimal multi-stage $\mathrm{CC}$ charging pattern. However, the obtained charging profile is fixed and cannot adapt to the operating condition in real time. In order to achieve higher charging efficiency, a FLC similar to that presented in [21] is integrated into the obtained five-stage CC charging algorithm to fine-tune the charging current. Figure 2 shows the block diagram of the proposed FLC. Because improper charging results in excessive temperature rise in the battery and temperature will also affect the effective charging resistance; therefore the temperature rise $T_{\mathrm{R}}$ and the gradient of temperature rise $\Delta T_{\mathrm{R}}$ are chosen as the inputs of the proposed FLC in this study. $T_{\mathrm{R}}$ and $\Delta T_{\mathrm{R}}$ can be defined as:

$$
\begin{gathered}
T_{R}(k)=T_{B}(k)-T_{A}(k) \\
\Delta T_{R}(k)=T_{R}(k)-T_{R}(k-1)
\end{gathered}
$$

where $T_{\mathrm{B}}$ is the battery surface temperature and $T_{\mathrm{A}}$ is the ambient temperature. 
Figure 2. Block diagram of the proposed FLC.

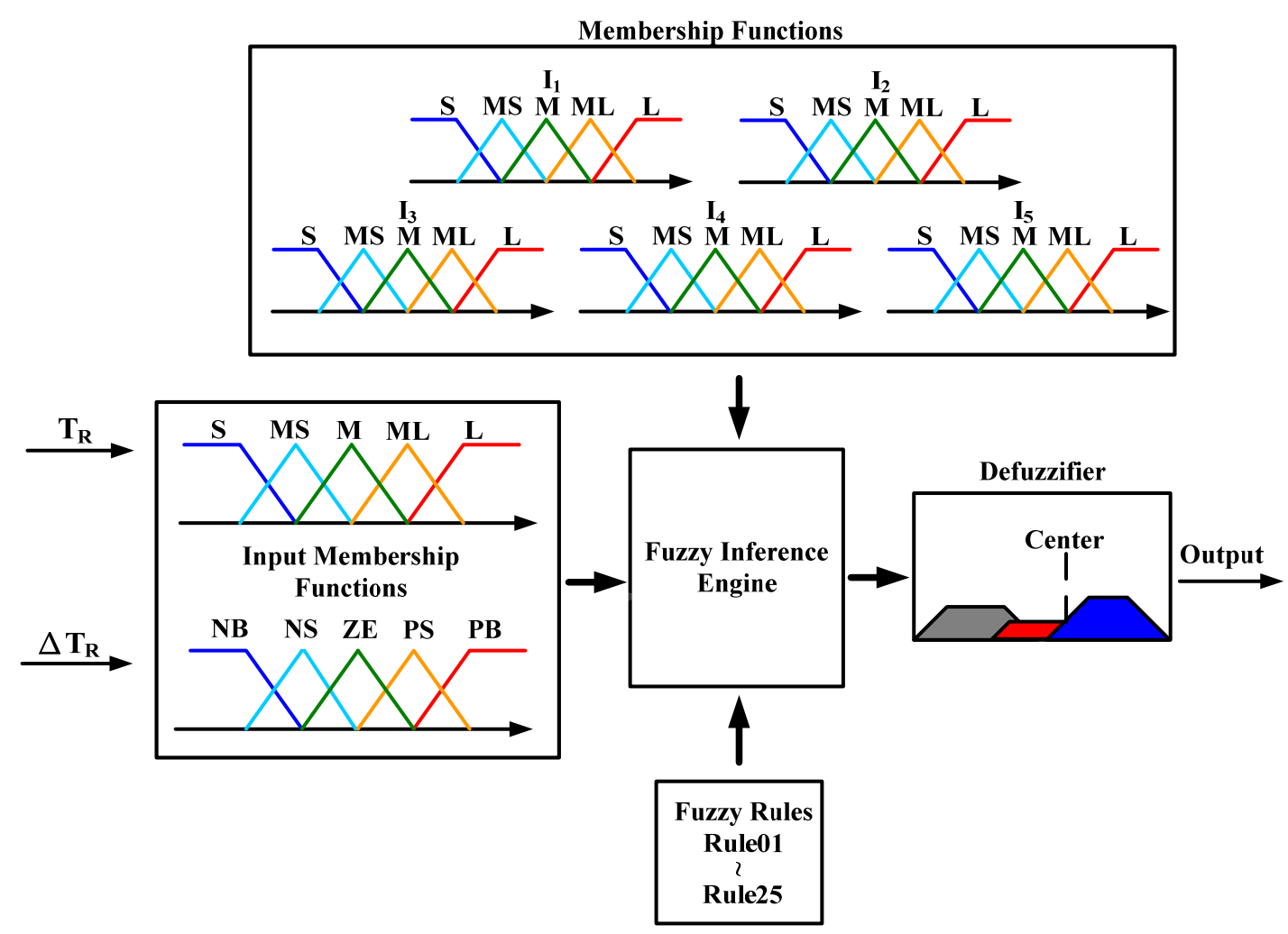

The MFs of the proposed FLC are shown in Figure 3. Figure 3a depicts the MF of the input variable $T_{\mathrm{R}}$, Figure $3 \mathrm{~b}$ illustrates the MF of the input variable $\Delta T_{\mathrm{R}}$ and Figure $3 \mathrm{c}$ displays the MFs of the output current command, respectively. Since the proposed FLC will be realized using a low cost DSC, triangular-shaped MFs are chosen to represent both the input and output variables so that the computation complexity can be reduced. It should be noted that the five-stage charging algorithm is employed in this study. Therefore, there are five sets of output MFs. In Figure 3, the linguistic variables $\mathrm{S}, \mathrm{MS}, \mathrm{M}, \mathrm{ML}, \mathrm{L}, \mathrm{NB}, \mathrm{NS}, \mathrm{ZE}, \mathrm{PS}$ and $\mathrm{PB}$, represents small, medium small, medium, medium large, large, negative big, negative small, zero, positive small and positive big, respectively. It should also be noted that the center value (i.e., $I_{X, \mathrm{M}}, x=1-5$ ) of the output MFs is set as the values equal to the optimal charging current values obtained in [17].

From Figure 3, each of the input variables $T_{\mathrm{R}}$ and $\Delta T_{\mathrm{R}}$ is mapped into five different linguistic values. Therefore, the rule base of the proposed FLC will contain 25 different rules. The basic principle of designing the rules is to adjust the charging current according to the temperature variation. The charging current will decrease when $T_{\mathrm{R}}$ or $\Delta T_{\mathrm{R}}$ increases, and vice versa. Based on this concept, the complete set of fuzzy control rules for the proposed system is tabulated in Table 1. The defuzzification method used in this study is the commonly used center of gravity (COG) method and is shown in Equation (3):

$$
y=\frac{\sum_{i=1}^{n} W_{i} B_{i}}{\sum_{i=1}^{n} W_{i}}
$$

where $W_{i}$ is the inference result of rule $I ; B_{i}$ is the corresponding output of rule $i$; and $y$ is the output. 
Figure 3. The MF definition of the proposed FLC (a) MF of the input variable $T_{\mathrm{R}}$; (b) MF of the input variable $\Delta T_{\mathrm{R}} ;$ (c) MFs of the output variable $I_{\mathrm{o}}$.

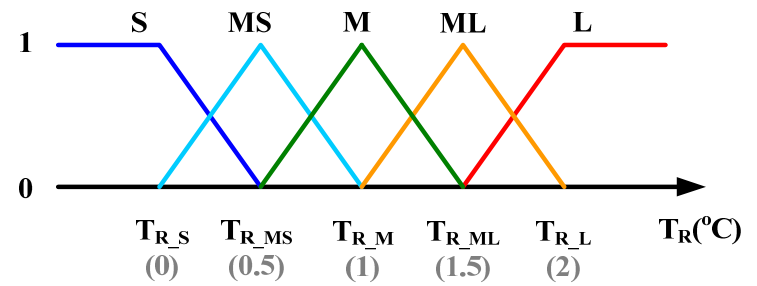

(a)
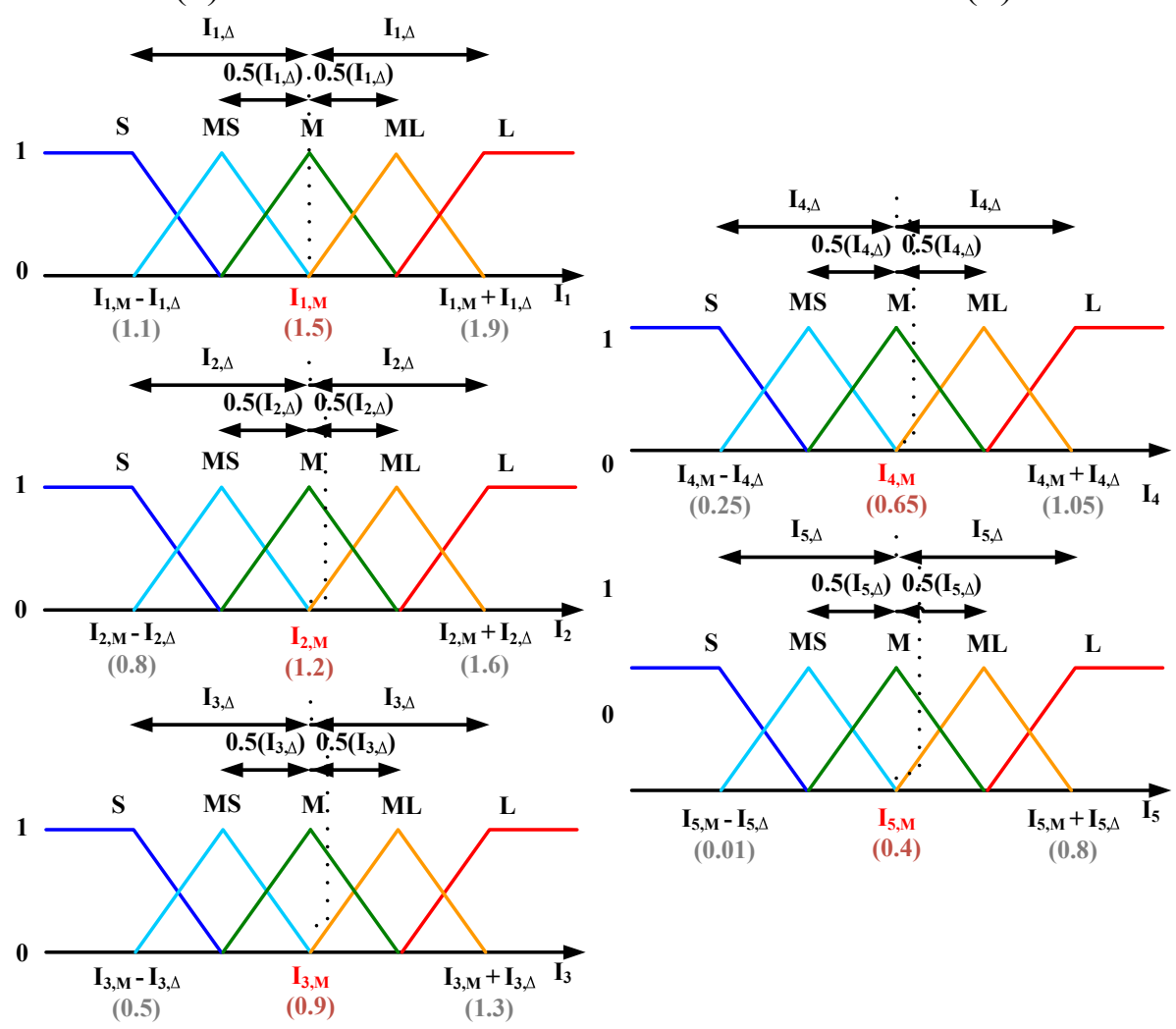

(c)

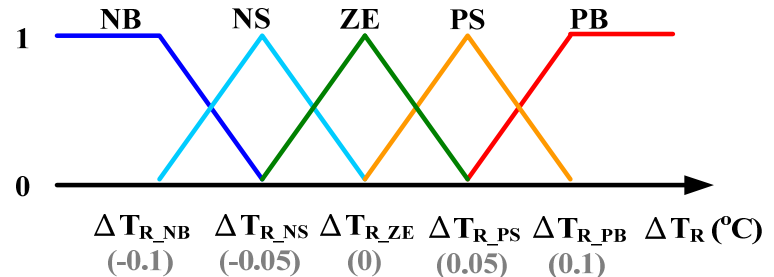

(b)

Table 1. Complete rule base for the proposed FLC.

\begin{tabular}{cccccc}
\hline \multirow{2}{*}{$\boldsymbol{T}_{\mathbf{R}}$} & \multicolumn{5}{c}{$\Delta \boldsymbol{T}_{\mathbf{R}}$} \\
\cline { 2 - 6 } & NB & NS & ZE & PS & PB \\
\hline \multirow{2}{*}{ S } & L & L & ML & ML & ML \\
& Rule1 & Rule2 & Rule3 & Rule4 & Rule5 \\
\hline \multirow{2}{*}{ MS } & L & ML & ML & M & M \\
& Rule6 & Rule7 & Rule8 & Rule9 & Rule10 \\
\hline \multirow{2}{*}{ M } & ML & ML & M & MS & MS \\
& Rule11 & Rule12 & Rule13 & Rule14 & Rule15 \\
\hline \multirow{2}{*}{ ML } & M & M & MS & MS & S \\
& Rule16 & Rule17 & Rule18 & Rule19 & Rule20 \\
\hline \multirow{2}{*}{ L } & MS & MS & MS & S & S \\
& Rule21 & Rule22 & Rule23 & Rule24 & Rule25 \\
\hline \multirow{2}{*yyyy}{}
\end{tabular}




\section{FLC Optimization Using Fuzzy-Based Taguchi Method}

Despite using the operator expertise and knowledge in the derivation of the control rules and the MFs of FLC, there are still some difficulties in determining the range of the MFs. To improve the proposed FLC, the fuzzy-based Taguchi method is utilized to determine the optimal output MFs. This is achieved according to the following procedures:

\section{a. Problem Description}

Both the input MFs and output MFs may affect the performance of the FLC; however, the attention is focused on the tuning of the output MF due to its stronger impact on the overall performance of the controller. From Figure 3c, the output MFs are defined by two parameters - the center value $I_{x, \mathrm{M}}$ and the adaption range $I_{x, \Delta}$. From Section 3 , the center value $I_{x, \mathrm{M}}$ should be equal to the optimal charging current values obtained in [17]. Therefore, $I_{x, \Delta}$ is chosen to be the target optimization parameter of this study. Figure 4 shows the experimental results of two FLC-based battery chargers (denoted as charger1 and charger-2). All the parameters of these two FLC-based battery chargers are identical, except for that charger-1 uses a larger $I_{x, \Delta}$ than charger-2. From the experimental results, the charging time is shorter but the charged capacity is lower for charger-1, and vice versa. To reiterate, a larger $I_{x, \Delta}$ leads to lower temperature rise but also results in lower charged capacity, while a smaller $I_{x, \Delta}$ increases the temperature rise and consequently impairs the charging efficiency. Therefore, a tradeoff between the charging efficiency and the charged capacity should be addressed.

Figure 4. Experiment results of two FLC-based battery controllers with different output MF settings.

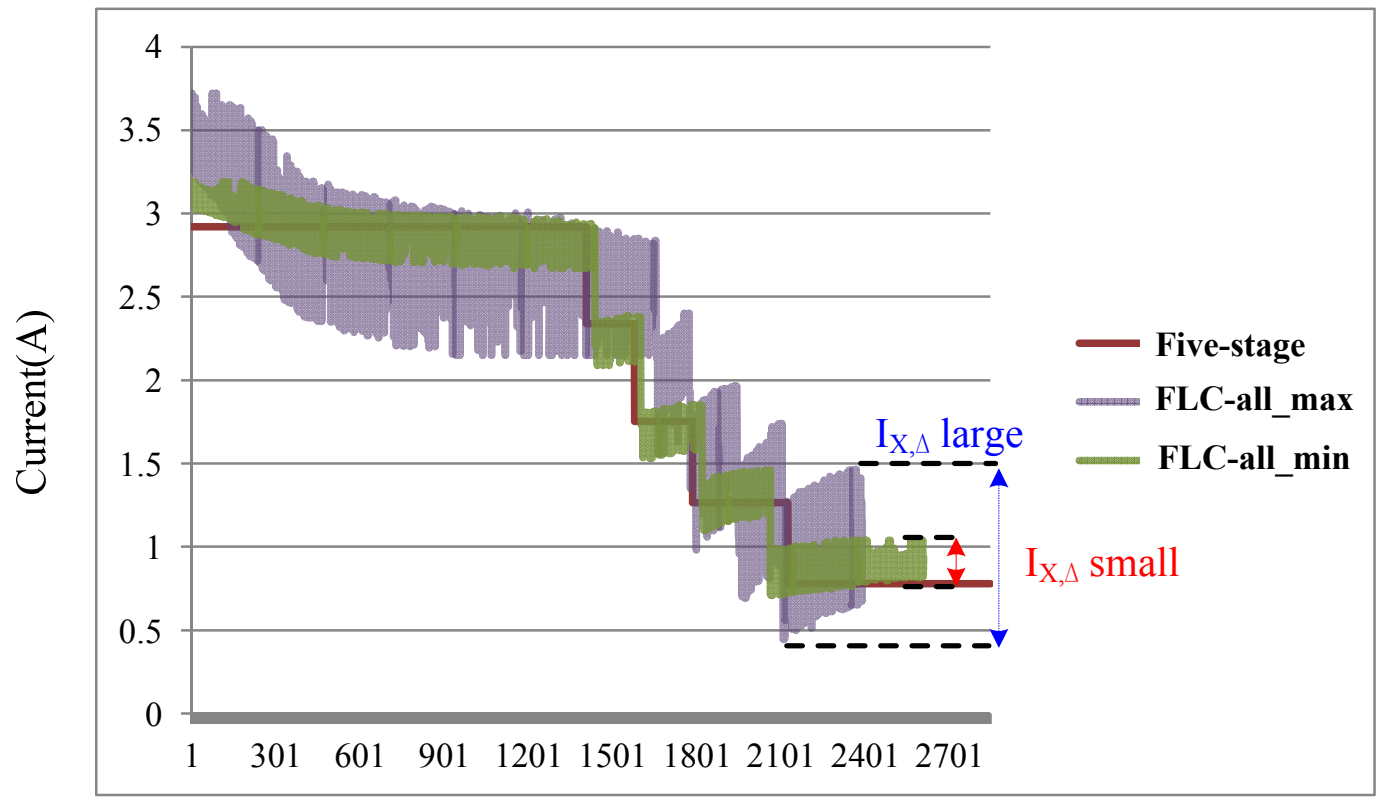

Time(sec) 


\section{b. Basic Concept of Taguchi Method}

For parameter optimization problems with a given cost function, the Taguchi method provides a simple, systematic, and efficient approach for obtaining the optimal parameter settings. The Taguchi method was developed on the basis of the orthogonal array (OA) concept. An OA can be built from the knowledge of the number of parameters and their levels. In general, the OA can be expressed by $\boldsymbol{L}_{\boldsymbol{B}}\left(\boldsymbol{C}^{\boldsymbol{D}}\right)$, where $\boldsymbol{B}, \boldsymbol{C}$, and $\boldsymbol{D}$ represent the number of experimental runs, the number of levels of each parameter, and the maximum number of parameters that the table can handle, respectively. In most cases, $\boldsymbol{B}$ is much smaller than the value of $\boldsymbol{C}^{\boldsymbol{D}}$, which is the total number of experiments required for $\boldsymbol{D}$ parameters having $\boldsymbol{C}$ levels. Consequently, the Taguchi method can dramatically reduce the number of necessary experiments by using OA. There are also mixed-level OA $\boldsymbol{L}_{\boldsymbol{B}}\left(\boldsymbol{C}^{\boldsymbol{D}} \times \boldsymbol{E}^{\boldsymbol{F}}\right)$, which can deal with $\boldsymbol{D}$ factors with $\boldsymbol{C}$ levels and $\boldsymbol{F}$ factors with $\boldsymbol{E}$ levels. Once the OA has been determined, experiments can be conducted accordingly, and the measured performance from each experiment can then be used to analyze the effect of different parameters. One of the key features of the Taguchi method is using the signal-to-noise ratio $(S / N$ ratio) to interpret the performance characteristic in the optimization process. The $S / N$ ratio can be calculated as:

$$
\begin{aligned}
& S / N=-10 \log \frac{S^{2}}{\bar{y}^{2}} \\
& \text { in which } \bar{y}=\frac{\sum_{i=1}^{n} y_{i}}{n} \text { and } S=\sqrt{\frac{\sum_{i=1}^{n}\left(y_{i}-\bar{y}\right)^{2}}{n-1}}
\end{aligned}
$$

where $y_{i}(i=1-n)$ represents the response values obtained at the $i$-th test; and $\mathrm{n}$ is the total number of tests for the same levels of parameters.

\section{c. Fuzzy-Based Taguchi Method with MPCI}

The goals of the proposed charger are to reduce charging time, increase charging efficiency and lessen the temperature rise simultaneously; therefore, it can be regarded as a multiple performance characteristic index (MPCI) problem. However, conventional Taguchi method can only find the optimal parameters based on a single performance characteristic. Hence, the fuzzy logic is combined with Taguchi method in this study to optimize the parameters $[29,30]$. In fuzzy-based Taguchi method, several fuzzy rules will be derived based on the performance requirement of the system. The $S / N$ ratio corresponding to each performance characteristic is fuzzified and then a combined performance index (CPI) is obtained through fuzzy reasoning. The CPI can then be used to optimize the target system.

\section{d. Application of Fuzzy-Based Taguchi Method to the Optimization of Output MF Parameter}

Figure 5 shows the implementation procedures of the utilized fuzzy-based Taguchi method. The steps of applying the fuzzy-based Taguchi method to optimize the proposed charger will be described in detail as follows: 


\section{Step 1. OA Design}

From the description above, $I_{x, \Delta}$ setting will affect the performance of the proposed charger. Therefore, an optimization of this parameter is required. Since there are five $I_{x, \Delta}$ setting values, consequently the number of the parameters is $5\left(I_{1, \Delta}-I_{5, \Delta}\right)$. Assuming that the levels for each parameter are chosen as 3 , a $\mathrm{L}_{18}\left(2^{1} \times 3^{7}\right) \mathrm{OA}$ can be selected to minimize the number of experiments required. The utilized $\mathrm{L}_{18}$ OA is shown in Table 2. In Table 2, numbers 1-3 stand for the high, middle, and low levels for each parameter, respectively. In this study, the setting values of these parameters are summarized in Table 3.

Table 2. $\mathrm{L}_{18}\left(2^{1} 3^{7}\right)$ Orthogonal array.

\begin{tabular}{ccccccccc}
\hline No. & $\mathbf{X}$ & $\boldsymbol{I}_{1}$ & $\boldsymbol{I}_{2}$ & $\boldsymbol{I}_{3}$ & $\boldsymbol{I}_{4}$ & $\boldsymbol{I}_{5}$ & $\mathbf{X}$ & $\mathbf{X}$ \\
\hline 1 & 1 & 1 & 1 & 1 & 1 & 1 & 1 & 1 \\
2 & 1 & 1 & 2 & 2 & 2 & 2 & 2 & 2 \\
3 & 1 & 1 & 3 & 3 & 3 & 3 & 3 & 3 \\
4 & 1 & 2 & 1 & 1 & 2 & 2 & 3 & 3 \\
5 & 1 & 2 & 2 & 2 & 3 & 3 & 2 & 1 \\
6 & 1 & 2 & 3 & 3 & 1 & 1 & 1 & 2 \\
7 & 1 & 3 & 1 & 2 & 1 & 3 & 2 & 3 \\
8 & 1 & 3 & 2 & 3 & 2 & 1 & 3 & 1 \\
9 & 1 & 3 & 3 & 1 & 3 & 2 & 1 & 2 \\
10 & 2 & 1 & 1 & 3 & 3 & 2 & 2 & 1 \\
11 & 2 & 1 & 2 & 1 & 1 & 3 & 3 & 2 \\
12 & 2 & 1 & 3 & 2 & 2 & 1 & 1 & 3 \\
13 & 2 & 2 & 1 & 2 & 3 & 1 & 3 & 2 \\
14 & 2 & 2 & 2 & 3 & 1 & 2 & 1 & 3 \\
15 & 2 & 2 & 3 & 1 & 2 & 3 & 2 & 1 \\
16 & 2 & 3 & 1 & 3 & 2 & 3 & 1 & 2 \\
17 & 2 & 3 & 2 & 1 & 3 & 1 & 2 & 3 \\
18 & 2 & 3 & 3 & 2 & 1 & 2 & 3 & 1 \\
\hline
\end{tabular}

Table 3. Setting values of the OA.

\begin{tabular}{cccccc}
\hline Level & $\boldsymbol{I}_{1, \mathbf{\Delta}}(\mathbf{C})$ & $\boldsymbol{I}_{2, \mathbf{\Delta}}(\mathbf{C})$ & $\boldsymbol{I}_{3, \mathbf{\Delta}}(\mathbf{C})$ & $\boldsymbol{I}_{\mathbf{4}, \mathbf{\Delta}}(\mathbf{C})$ & $\boldsymbol{I}_{5, \mathbf{\Delta}}(\mathbf{C})$ \\
\hline $\mathrm{H}(1)$ & 0.4 & 0.4 & 0.4 & 0.4 & 0.4 \\
$\mathrm{M}(2)$ & 0.267 & 0.267 & 0.267 & 0.267 & 0.267 \\
$\mathrm{~L}(3)$ & 0.133 & 0.133 & 0.133 & 0.133 & 0.133 \\
\hline
\end{tabular}

Step 2. Conduct the Experiment

The experiments are carried out utilizing an implemented FLC-based battery charger as shown in Figure 1. The output MF parameter is read from the designed OA and is programmed into the firmware of the proposed charger. After charging the batteries, the discharging processes are then carried out using a WBCS3000 battery cycle life tester (5 A, $5 \mathrm{~V}$, and eight channels) from WonATech Corp. Because large discharging current tends to degrade the discharging efficiency, a small discharging current of $\mathrm{C} / 10$ is utilized to obtain a more accurate total charged capacity value. The 
obtained charged capacity, charging time and temperature rise are then recorded for performance evaluation. In this study, experimental trials corresponding to the same OA levels will be performed three times.

\section{Step 3. Design of the Fuzzy Logic Unit}

The objective of the proposed charger is to find a compromise between the charged capacity and the charging time. Therefore, the performance index should take the charged capacity, the charging time and the temperature rise into account. In this study, a three-input-one-output fuzzy logic unit (FLU) is employed to combine these three performance characteristics into a single performance index, as shown in Figure 5.

Figure 5. Implementation procedures of the utilized fuzzy-based Taguchi method.

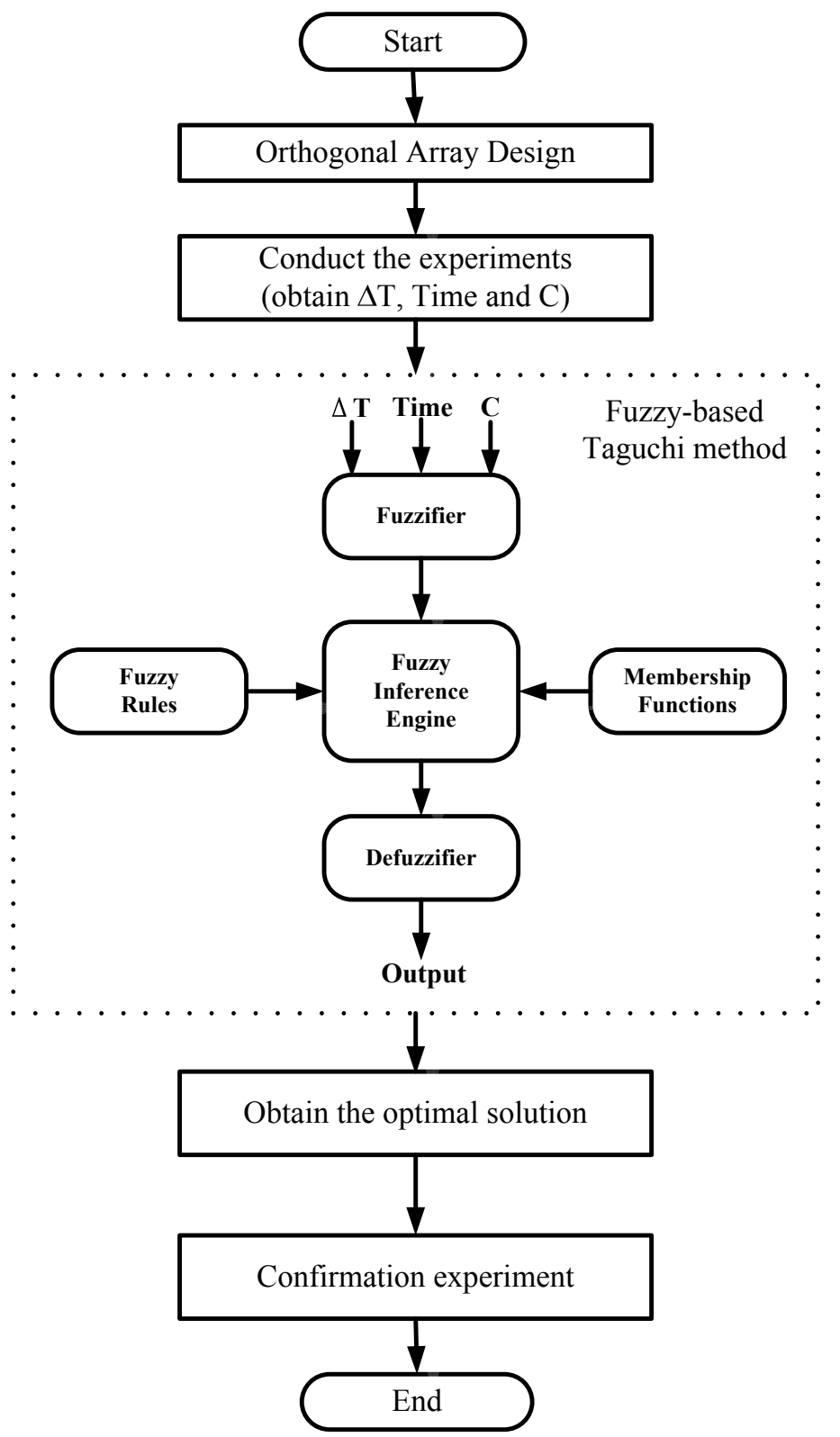


From Figure 5, the inputs of the designed FLU are the charged capacity, charging time and the temperature rise. Figure 6 shows the input MFs of the proposed FLU. From Figure 6, each of the input MFs can be defined by five linguistic variables, the values of these variables can be calculated as:

$$
\begin{gathered}
D C=\text { Max }- \text { Min } \\
L=\text { Max }+D C * 0.2 \\
S=\text { Min }-D C * 0.2 \\
M=\frac{L+S}{2} \\
M S=\frac{S+M}{2} \\
M L=\frac{M+L}{2}
\end{gathered}
$$

where Max and Min corresponds to the maximum and minimum obtained value for each input variables.

Figure 6. Input MFs of the proposed FLU (a) Input membership function for the charged capacity; (b) Input membership function for charging time; (c) Input membership function for temperature rise.

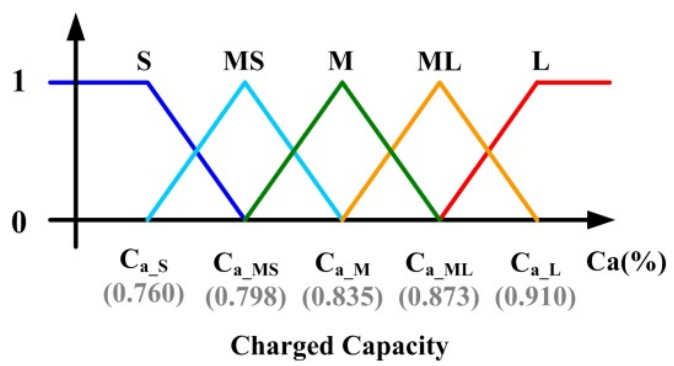

(a)

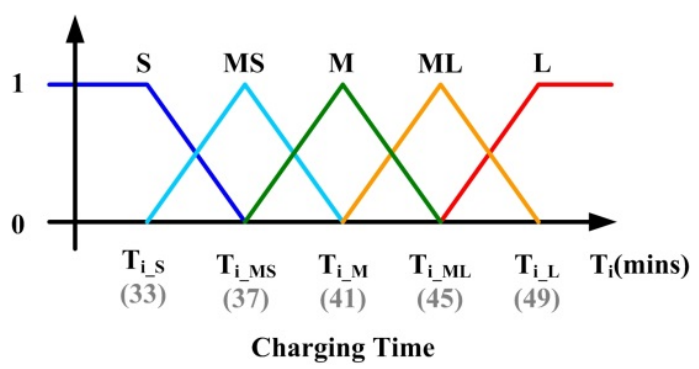

(b)

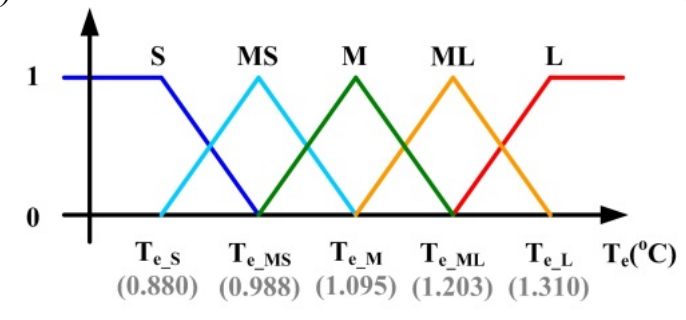

Temperature rise

(c)

The output MF of the proposed FLU is shown in Figure 7. To increase the resolution, nine linguistic variables are utilized to define the output MF, these linguistic variables are evenly distributed in the $[0,1]$ interval. In this study, triangular MFs are chosen to represent both the input and output variables. In Figures 6 and 7, the linguistic variables VS, SS, S, MS, M, ML, L, LL and VL represents extremely small, very small, small, medium small, medium, medium large, large, very large and extremely large, respectively. 
Figure 7. Output MF of of the proposed FLU.

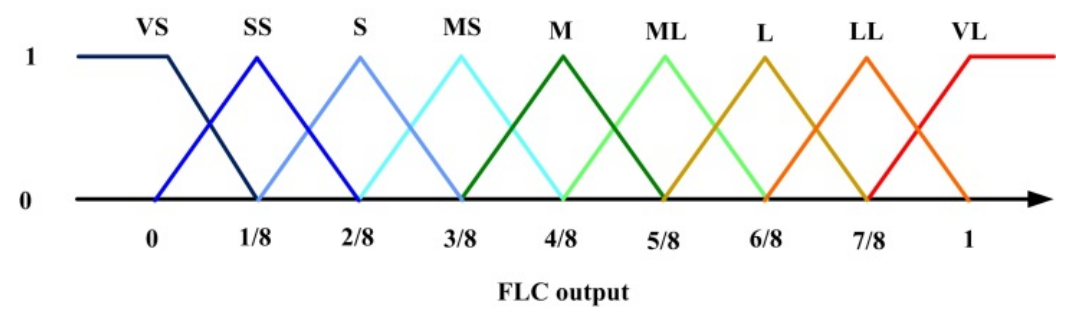

From Figure 6, there are three input variables and each of the input variables is mapped into five different linguistic values. Therefore, the rule base of the proposed FLU will contain $5 \times 25$ different rules. The basic principle for designing the rules is that the performance index will be larger for higher charged capacity value, lower charging time and lower temperature rise. Based on this concept, the complete sets of fuzzy rules for the proposed FLU are summarized in Tables 4-8. The defuzzification method used in the proposed FLU is also the COG method.

Table 4. Rule base of the proposed FLU for $\boldsymbol{T}_{\mathbf{e}}=\mathrm{S}$.

\begin{tabular}{cccccc}
\hline \multirow{2}{*}{$\boldsymbol{T}_{\mathbf{i}}$} & \multicolumn{5}{c}{ Ca } \\
\cline { 2 - 6 } S & S & MS & M & ML & L \\
\hline \multirow{2}{*}{ S } & L & LL & VL & VL & VL \\
& Rule1 & Rule2 & Rule3 & Rule4 & Rule5 \\
\hline \multirow{2}{*}{ MS } & ML & L & LL & VL & VL \\
& Rule6 & Rule7 & Rule8 & Rule9 & Rule10 \\
\hline \multirow{2}{*}{ M } & M & ML & L & LL & VL \\
& Rule11 & Rule12 & Rule13 & Rule14 & Rule15 \\
\hline \multirow{2}{*}{ ML } & MS & M & ML & L & LL \\
& Rule16 & Rule17 & Rule18 & Rule19 & Rule20 \\
\hline \multirow{2}{*}{ L } & S & MS & M & ML & L \\
& Rule21 & Rule22 & Rule23 & Rule24 & Rule25 \\
\hline
\end{tabular}

Table 5. Rule base of the proposed FLU for $\boldsymbol{T}_{\mathbf{e}}=$ MS.

\begin{tabular}{cccccc}
\hline \multirow{2}{*}{$\boldsymbol{T}_{\mathbf{i}}$} & \multicolumn{5}{c}{ Ca } \\
\cline { 2 - 6 } & S & MS & M & ML & L \\
\hline \multirow{2}{*}{ S } & ML & L & LL & VL & VL \\
& Rule1 & Rule2 & Rule3 & Rule4 & Rule5 \\
\hline \multirow{2}{*}{ MS } & M & ML & L & LL & VL \\
& Rule6 & Rule7 & Rule8 & Rule9 & Rule10 \\
\hline \multirow{2}{*}{ M } & MS & M & ML & L & LL \\
& Rule11 & Rule12 & Rule13 & Rule14 & Rule15 \\
\hline \multirow{2}{*}{ ML } & S & MS & M & ML & L \\
& Rule16 & Rule17 & Rule18 & Rule19 & Rule20 \\
\hline \multirow{2}{*}{ L } & SS & S & MS & M & ML \\
& Rule21 & Rule22 & Rule23 & Rule24 & Rule25 \\
\hline
\end{tabular}


Table 6. Rule base of the proposed FLU for $\boldsymbol{T}_{\mathbf{e}}=\mathrm{M}$.

\begin{tabular}{cccccc}
\hline \multirow{2}{*}{$\boldsymbol{T}_{\mathbf{i}}$} & \multicolumn{5}{c}{ Ca } \\
\cline { 2 - 6 } S & S & MS & M & ML & L \\
\hline \multirow{2}{*}{ S } & $\begin{array}{c}\text { M } \\
\text { Rule1 }\end{array}$ & $\begin{array}{c}\text { Rule2 } \\
\text { MS }\end{array}$ & $\begin{array}{c}\text { L } \\
\text { Rule3 }\end{array}$ & $\begin{array}{c}\text { LL } \\
\text { Rule4 }\end{array}$ & $\begin{array}{c}\text { VL } \\
\text { Rule5 }\end{array}$ \\
\hline \multirow{2}{*}{ Mule6 } & Rule7 & Rule8 & Rule9 & Rule10 \\
\hline \multirow{2}{*}{ M } & S & MS & M & ML & L \\
& Rule11 & Rule12 & Rule13 & Rule14 & Rule15 \\
\hline \multirow{2}{*}{ ML } & SS & S & MS & M & ML \\
& Rule16 & Rule17 & Rule18 & Rule19 & Rule20 \\
\hline \multirow{2}{*}{ L } & VS & SS & S & MS & M \\
& Rule21 & Rule22 & Rule23 & Rule24 & Rule25 \\
\hline
\end{tabular}

Table 7. Rule base of the proposed FLU for $\boldsymbol{T}_{\mathrm{e}}=$ ML.

\begin{tabular}{cccccc}
\hline \multirow{2}{*}{$\boldsymbol{T}_{\mathbf{i}}$} & \multicolumn{5}{c}{ Ca } \\
\cline { 2 - 6 } & S & MS & M & ML & L \\
\hline \multirow{2}{*}{ S } & MS & M & ML & L & LL \\
& Rule1 & Rule2 & Rule3 & Rule4 & Rule5 \\
\hline \multirow{2}{*}{ MS } & S & MS & M & ML & L \\
& Rule6 & Rule7 & Rule8 & Rule9 & Rule10 \\
\hline \multirow{2}{*}{ M } & SS & S & MS & M & ML \\
& Rule11 & Rule12 & Rule13 & Rule14 & Rule15 \\
\hline \multirow{2}{*}{ ML } & VS & SS & S & MS & M \\
& Rule16 & Rule17 & Rule18 & Rule19 & Rule20 \\
\hline \multirow{2}{*}{ L } & VS & VS & SS & S & MS \\
& Rule21 & Rule22 & Rule23 & Rule24 & Rule25 \\
\hline
\end{tabular}

Table 8. Rule base of the proposed FLU for $\boldsymbol{T}_{\mathbf{e}}=\mathrm{L}$.

\begin{tabular}{cccccc}
\hline \multirow{2}{*}{$\boldsymbol{T}_{\mathbf{i}}$} & \multicolumn{5}{c}{ Ca } \\
\cline { 2 - 6 } & S & MS & M & ML & L \\
\hline \multirow{2}{*}{ S } & S & MS & M & ML & L \\
& Rule1 & Rule2 & Rule3 & Rule4 & Rule5 \\
\hline \multirow{2}{*}{ MS } & SS & S & MS & M & ML \\
& Rule6 & Rule7 & Rule8 & Rule9 & Rule10 \\
\hline \multirow{2}{*}{ M } & VS & SS & S & MS & M \\
& Rule11 & Rule12 & Rule13 & Rule14 & Rule15 \\
\hline \multirow{2}{*}{ ML } & VS & VS & SS & S & MS \\
& Rule16 & Rule17 & Rule18 & Rule19 & Rule20 \\
\hline \multirow{2}{*}{ L } & VS & VS & VS & SS & S \\
& Rule21 & Rule22 & Rule23 & Rule24 & Rule25 \\
\hline
\end{tabular}


Step 4. Obtaining the Optimal Solution Through Taguchi Approach

In Step 3, a FLU is proposed to combine three performance characteristics into a single performance index. In this study, the output of the FLU is called CPI. Based on the above discussion, the performance of the charger is better if the obtained CPI is larger. Since the experimental design is orthogonal, it is then possible to separate out the effect of each parameter at different levels and determine the optimal combinations of the output MF parameter levels.

Step 5. Performing Confirmation Tests

Once the optimal level of the parameters is obtained, confirmation experiments under these conditions will be performed to compare the results with the predictions. If the results agree with the predictions, the obtained results can be regarded as valid. Otherwise, the above steps must be repeated.

\section{Experimental Results}

From Section 4, the designed fuzzy-based Taguchi method utilizes a $\mathrm{L}_{18} \mathrm{OA}$ to obtain the optimal setting values of $I_{x, \Delta}$ After performing the experiments, the measured results are summarized in Table 9. Based on the data shown in Table 9, the average $S / N$ ratio responses of each parameter can be calculated at each level.

Table 9. Measured results for the $\mathrm{L}_{18} \mathrm{OA}$.

\begin{tabular}{ccccccc}
\hline No. & Fuzzy Results $_{1}$ & Fuzzy Results $_{\mathbf{2}}$ & Fuzzy Results $_{\mathbf{3}}$ & y(Avg.) & $\boldsymbol{S}$ & $\boldsymbol{S} / \boldsymbol{N}$ \\
\hline 1 & 0.274 & 0.429 & 0.478 & 0.3937 & 0.1065 & 11.356 \\
2 & 0.407 & 0.485 & 0.484 & 0.4587 & 0.0447 & 20.215 \\
3 & 0.437 & 0.548 & 0.552 & 0.5123 & 0.0653 & 17.897 \\
4 & 0.420 & 0.539 & 0.421 & 0.4600 & 0.0684 & 16.552 \\
5 & 0.402 & 0.553 & 0.554 & 0.5030 & 0.0875 & 15.194 \\
6 & 0.363 & 0.439 & 0.534 & 0.4453 & 0.0857 & 14.317 \\
7 & 0.456 & 0.517 & 0.556 & 0.5097 & 0.0504 & 20.097 \\
8 & 0.436 & 0.467 & 0.552 & 0.4850 & 0.0601 & 18.143 \\
9 & 0.457 & 0.573 & 0.588 & 0.5393 & 0.0717 & 17.527 \\
10 & 0.478 & 0.538 & 0.594 & 0.5367 & 0.0580 & 19.324 \\
11 & 0.493 & 0.548 & 0.647 & 0.5627 & 0.0780 & 17.159 \\
12 & 0.402 & 0.436 & 0.592 & 0.4767 & 0.1013 & 13.451 \\
13 & 0.396 & 0.457 & 0.601 & 0.4847 & 0.1053 & 13.263 \\
14 & 0.445 & 0.434 & 0.574 & 0.4843 & 0.0778 & 15.878 \\
15 & 0.444 & 0.626 & 0.612 & 0.5607 & 0.1013 & 14.864 \\
16 & 0.479 & 0.496 & 0.649 & 0.5413 & 0.0936 & 15.241 \\
17 & 0.360 & 0.429 & 0.589 & 0.4593 & 0.1175 & 11.844 \\
18 & 0.408 & 0.451 & 0.594 & 0.4843 & 0.0974 & 13.934 \\
C.E & 0.556 & 0.632 & 0.672 & 0.6200 & 0.0589 & 20.442 \\
\hline
\end{tabular}

Table 10 shows the response table and Figure 8 shows the response graph for the $S / N$ ratio. Since larger $S / N$ ratio corresponds to better performance characteristic, it can be seen that the factor-level combination $\left[I_{1, \Delta}(1), I_{2, \Delta}(2), I_{3, \Delta}(3), I_{4, \Delta}(2), I_{5, \Delta}(2)\right]$ contributes to maximize the performance index. 
Since this combination does not exist in the original $\mathrm{L}_{18} \mathrm{OA}$, confirmation test should be conducted to verify the correctness of this optimization procedure. The results of the confirmation test using the optimal parameters are shown in the last line of Table 9.

Table 10. Response table for the $S / N$ ratio.

\begin{tabular}{ccccccccc}
\hline $\boldsymbol{S} / \boldsymbol{N}$ & $\mathbf{X}$ & $\boldsymbol{I}_{1}$ & $\boldsymbol{I}_{2}$ & $\boldsymbol{I}_{3}$ & $\boldsymbol{I}_{4}$ & $\boldsymbol{I}_{5}$ & $\mathbf{X}$ & $\mathbf{X}$ \\
\hline Level1 & - & 16.57 & 15.97 & 14.88 & 15.46 & 13.73 & - & - \\
Level2 & - & 15.74 & 16.41 & 16.03 & 16.41 & 17.24 & - & - \\
Level3 & - & 16.13 & 15.33 & 16.80 & 15.84 & 16.74 & - & - \\
$\mathrm{E}_{1-2}$ & - & $(0.83)$ & 0.43 & 1.14 & 0.95 & 3.51 & - & - \\
$\mathrm{E}_{2-3}$ & - & 0.39 & $(1.07)$ & 0.77 & $(0.57)$ & $(0.50)$ & - & - \\
Range & - & 0.83 & 1.07 & 1.92 & 0.95 & 3.51 & - & - \\
Rank & - & 5 & 3 & 2 & 4 & 1 & - & - \\
\hline
\end{tabular}

Figure 8. Response graph for the $S / N$ ratio.

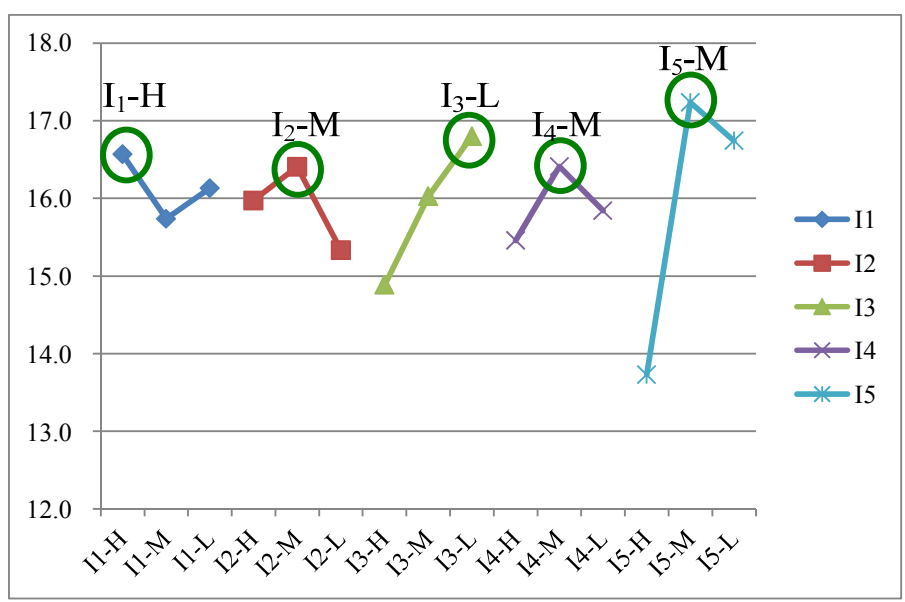

In order to verify the performance of the obtained optimal parameter setting, several experiments are also carried out. Figure 9 shows the measured temperature rise of the $\mathrm{CC}-\mathrm{CV}$ method and the utilized five-stage CC charging algorithm.

Figure 9. Temperature rise of the five-stage $\mathrm{CC}$ charging algorithm and the conventional CC-CV algorithm.

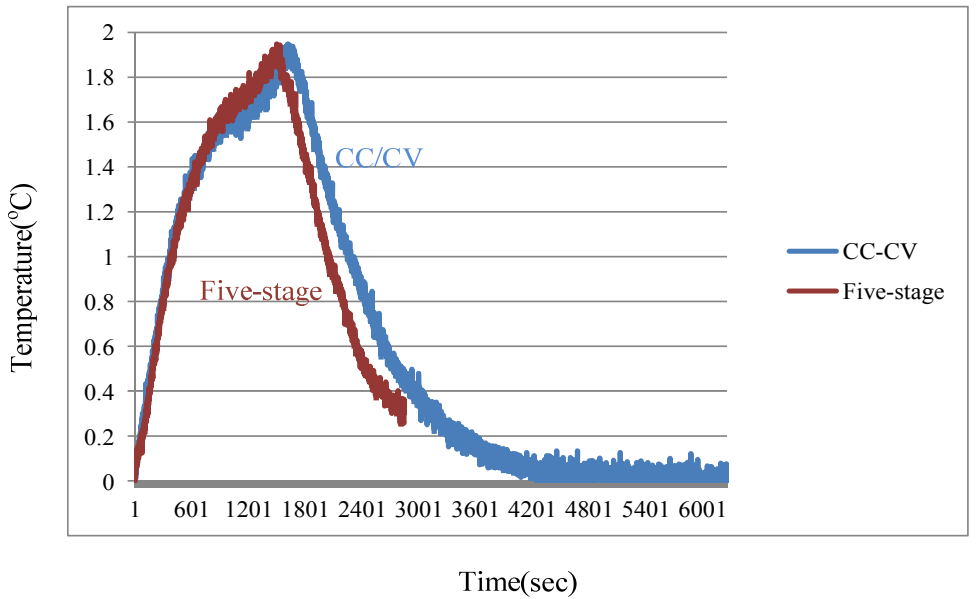


From Figure 9, the temperature rise of the utilized five-stage $\mathrm{CC}$ charging algorithm is lower than that of the conventional $\mathrm{CC}-\mathrm{CV}$ method. Therefore, the charging efficiency of the five-stage $\mathrm{CC}$ charging algorithm is higher. Similarly, Figure 10 shows the measured temperature rise of the utilized five-stage CC charging algorithm and two FLC-based charging algorithms with different output MF settings.

Figure 10. Temperature rise of the five-stage $\mathrm{CC}$ charging algorithm and the proposed FLC-based charging algorithm.

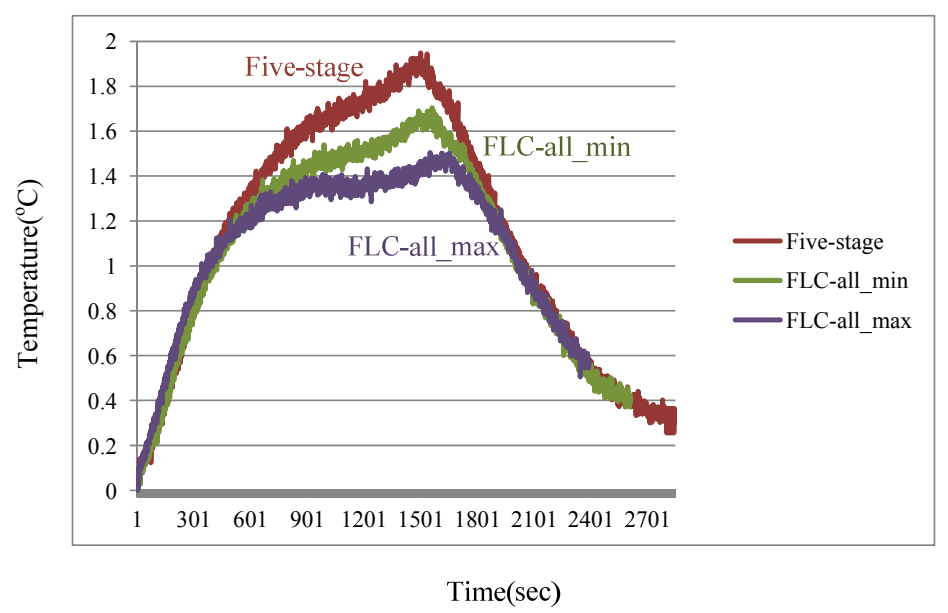

In Figure 10, the curve denoted as "FLC-all_min" is the FLC-based battery charger with all the $I_{x, \Delta}$ parameters set as low level $\left[I_{1, \Delta}(3), I_{2, \Delta}(3), I_{3, \Delta}(3), I_{4, \Delta}(3), I_{5, \Delta}(3)\right]$, and the curve denoted as "FLC-all_max" is the charger with all the $I_{x, \Delta}$ parameters set as high level $\left[I_{1, \Delta}(1), I_{2, \Delta}(1), I_{3, \Delta}(1)\right.$, $\left.I_{4, \Delta}(1), I_{5, \Delta}(1)\right]$. From Figure 10, the proposed FLC-based battery charger has higher charging efficiency than the five-stage $\mathrm{CC}$ charging algorithm regardless of the parameters setting value. Figure 11 shows the measured temperature rise of the proposed FLC-based battery charger with different settings.

Figure 11. Temperature rise of the proposed FLC-based charging algorithm with different parameter settings.

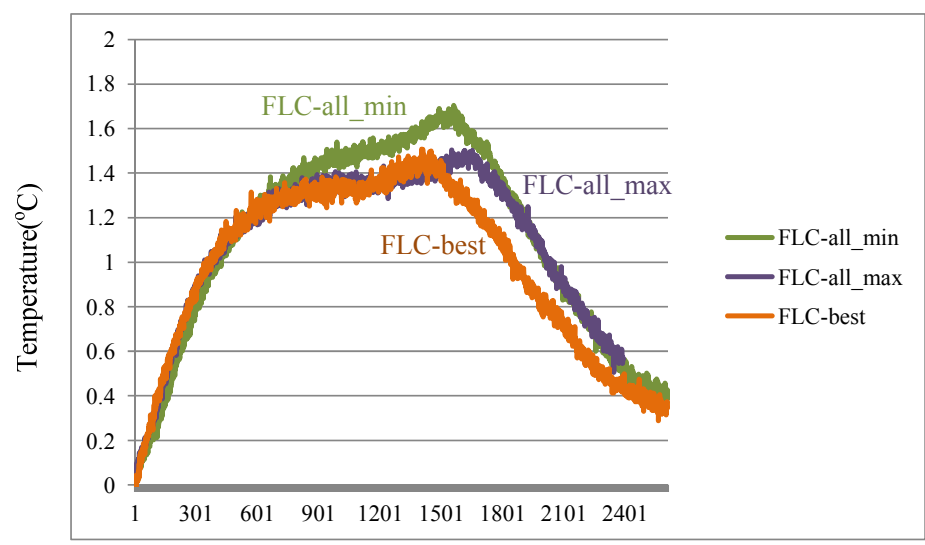

Time(sec)

In Figure 11, the curve denoted as "FLC-best" is the FLC-based battery charger with the optimal parameter setting $\left[I_{1, \Delta}(1), I_{2, \Delta}(2), I_{3, \Delta}(3), I_{4, \Delta}(2), I_{5, \Delta}(2)\right]$. From Figure 11, the FLC-based battery 
charger with the optimal parameter setting has the lowest temperature rise. In order to provide clearer overall comparison, temperature rise data of all the tested algorithms are again illustrated in Figure 12.

Figure 12. Temperature rise of five tested algorithms.

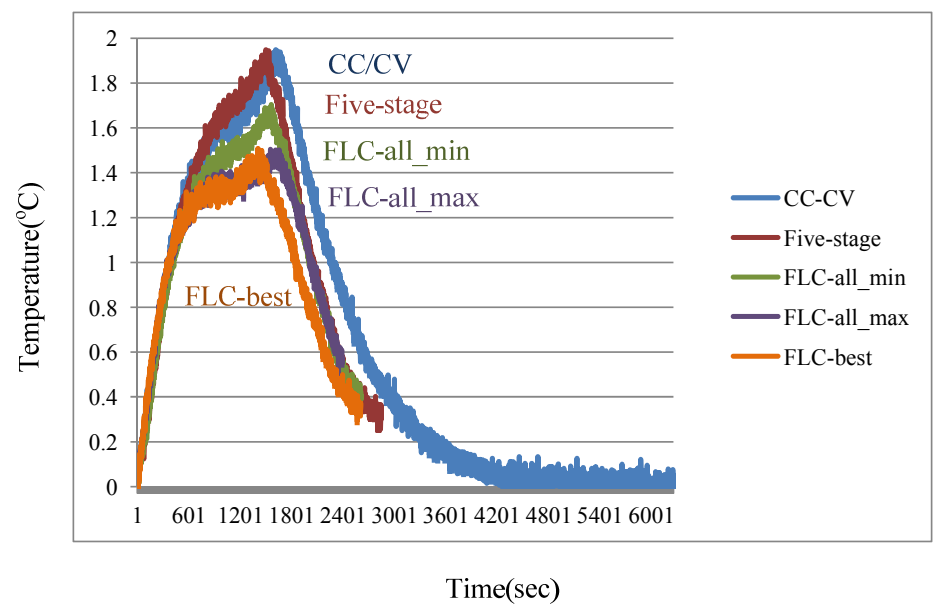

To further validate the effectiveness of the proposed charger, additional experiments are carried out. Table 11 and Figure 13 show the calculated MCPI results obtained from charging three brand-new batteries using five different charging algorithms. From Figure 13, the proposed FLC-based battery charger with optimal parameter setting has the best MCPI value for all the three sample batteries. Table 12 summarizes the charging efficiency, the average $\Delta \mathrm{T}$, the charged capacity, the charging time required and the combined MCPI value for five different charging patterns. In Table 12, the charging efficiency is defined as the discharged capacity divided by the charged capacity, and the average $\Delta \mathrm{T}$ is defined as the integration of the temperature rise for the whole charging period (the area under the temperature curve) divided by the charging time required. From Table 12, although the discharged capacity of the proposed FLC-based charger is lower than that of the CC-CV method, the charging time required can significantly be improved. Figure 14 illustrates the remaining capacity (in \%) versus cycle numbers for two new batteries from the same manufacturer (2000 mAh), charged using the $\mathrm{CC}-\mathrm{CV}$ charging method and the proposed charger. From Figure 14, the cycling number of the proposed algorithm when the capacity drop to $93.3 \%$ of the original capacity is 118 ; the cycling number of the conventional CC-CV method is 48 for the same capacity drop. That is, the proposed charge algorithm can provide $59.3 \%$ more cycle-life count than that of the conventional $\mathrm{CC}-\mathrm{CV}$ method. Comparing with the $\mathrm{CC}-\mathrm{CV}$ method, the charging time, charging efficiency, average temperature rise and the obtained cycle life of the Li-ion battery are improved by about $58.3 \%$, $1.65 \%, 26.7 \%$ and $59.3 \%$, respectively. Comparing with the five-stage CC algorithm, adding the FLC to fine-tune the current can improve the charging time, the charging efficiency and the average temperature rise of the Li-ion battery by about $8.35 \%, 0.99 \%$ and $20.3 \%$, respectively.

Table 11. Obtained MCPI results of five tested algorithms.

\begin{tabular}{cccccc}
\hline MCPI_results & CC-CV & Five-stage & FLC all_min & FLC all_max & FLC-best \\
\hline Sample1 & 0.250 & 0.253 & 0.265 & 0.274 & 0.556 \\
Sample2 & 0.250 & 0.280 & 0.361 & 0.429 & 0.632 \\
Sample3 & 0.281 & 0.348 & 0.421 & 0.478 & 0.672 \\
\hline
\end{tabular}


Table 12. Comparison of five tested algorithms.

\begin{tabular}{cccccc}
\hline Performance & CC-CV & Five-stage & FLC all_min & FLC all_max & FLC-best \\
\hline Charging efficiency $(\%)$ & 97.50 & 98.16 & 98.64 & 98.81 & 99.15 \\
Charging avg. $\Delta \mathrm{T}\left({ }^{\circ} \mathrm{C}\right)$ & 1.3326 & 1.2257 & 1.1840 & 1.1778 & 0.9771 \\
Charging time $(\mathrm{sec})$ & 6268 & 2852 & 2616 & 2395 & 2614 \\
Discharged cap. $(\%)$ & 100 & 87.65 & 84.10 & 81.89 & 85.02 \\
MCPI_results & 0.250 & 0.284 & 0.364 & 0.401 & 0.625 \\
\hline
\end{tabular}

Figure 13. Obtained MCPI results of five tested algorithms.

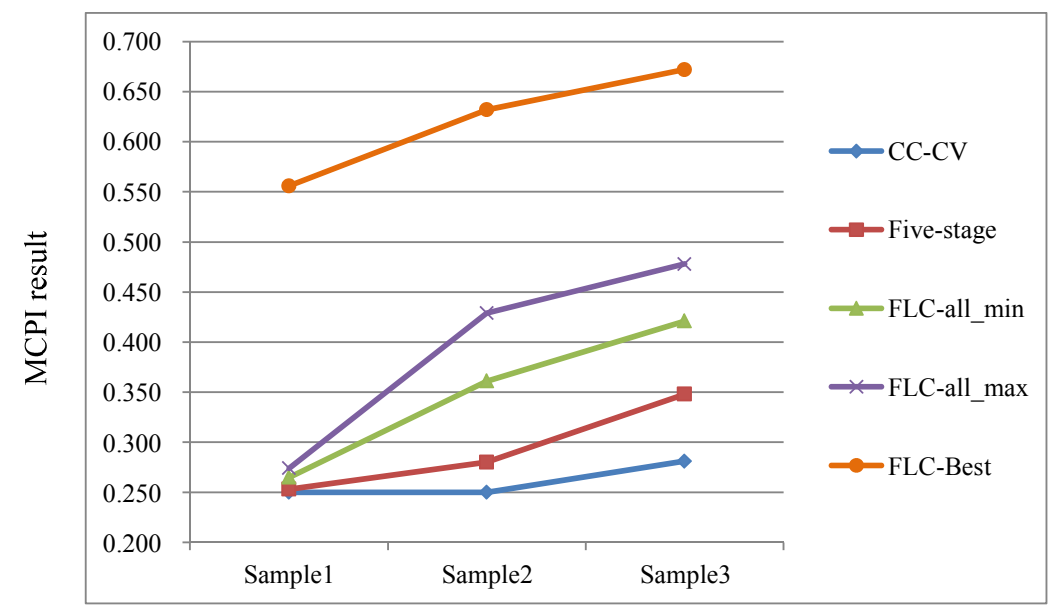

Figure 14. Comparison of cycle life test between the proposed charger and the CC-CV method.

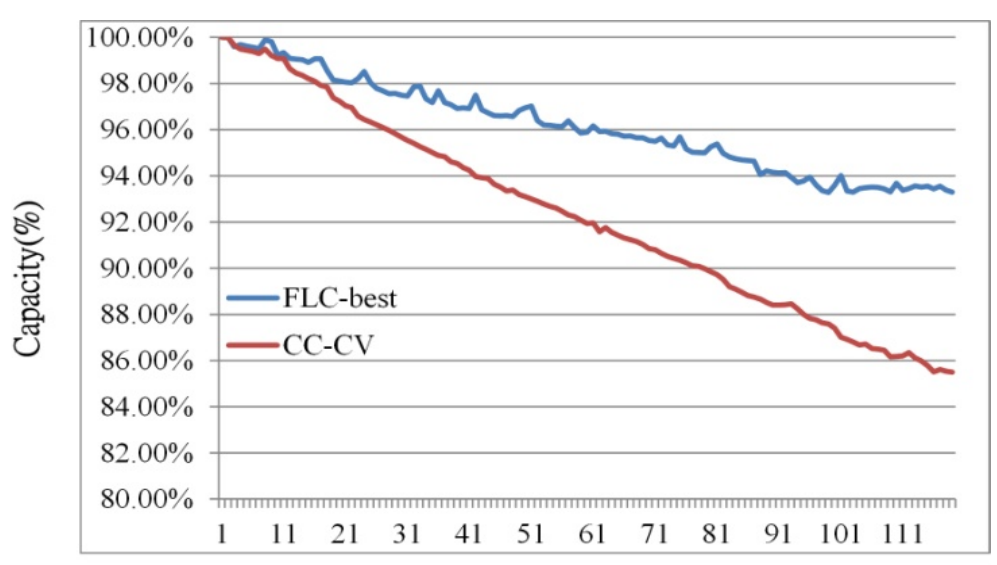

Cycle no.

\section{Conclusions}

In this study, a FLC-based five-stage Li-ion battery charger is first proposed. Based on an obtained optimal five-stage charging current setting value, the proposed charger integrates a FLC to fine-tune the charging current according to the battery temperature. Comparing with the conventional CC-CV and five-stage $\mathrm{CC}$ charging methods, the proposed charger is capable of charging the Li-ion batteries with shorter charging time, higher charging efficiency and lower temperature rise. To further improve the performance of the proposed charger, a fuzzy-based Taguchi method is utilized. The fuzzy-based Taguchi method can obtain the optimal output MFs in only 54 experimental tests. According to the 
experimental results, the performance characteristics such as the charging time, the charged capacity and the temperature rise can simultaneously be considered and improved through this approach. Comparing with the conventional CC-CV method, the charging time, the charging efficiency, average temperature rise and the obtained cycle life of the Li-ion battery are improved by about $58.3 \%, 1.65 \%$, $26.7 \%$ and $59.3 \%$, respectively.

\section{References}

1. Yan, J.; Xu, G.; Qian, H.; Xu, Y.; Song, Z. Model predictive control-based fast charging for vehicular batteries. Energies 2011, 4, 1178-1196.

2. Omar, N.; Daowd, M.; Bossche, P.; Hegazy, O.; Smekens, J.; Coosemans, T.; Mierlo, J. Rechargeable energy storage systems for plug-in hybrid electric vehicles-Assessment of electrical characteristics. Energies 2012, 5, 2952-2988.

3. Shuaib, K.; Zhang, L.; Gaouda, A.; Abdel-Hafez, M. A PEV charging service model for smart grids. Energies 2012, 5, 4665-4682.

4. Nguyen, M.; Nguyen, D.; Yoon, Y. A new battery energy storage charging/discharging scheme for wind power producers in real-time markets. Energies 2012, 5, 5439-5452.

5. Shiau, J.; Ma, C. Li-ion battery charging with a buck-boost power converter for a solar powered battery management system. Energies 2013, 6, 1669-1699.

6. Chen, L.R. PLL-based battery charge circuit topology. IEEE Trans. Ind. Electron. 2004, 51, 1344-1346.

7. Chen, L.R.; Chen, J.J.; Chu, N.Y.; Han, G.Y. Current-pumped battery charger. IEEE Trans. Ind. Electron. 2008, 55, 2482-2488.

8. Chen, L.R. A design of an optimal battery pulse charge system by frequency-varied technique. IEEE Trans. Ind. Electron. 2007, 54, 398-405.

9. Chen, L.R. Design of duty-varied voltage pulse charger for improving Li-ion battery-charging response. IEEE Trans. Ind. Electron. 2009, 56, 480-487.

10. Chen, L.R.; Liu, C.S.; Chen, J.J. Improving phase-locked battery charger speed by using resistance-compensated technique. IEEE Trans. Ind. Electron. 2009, 56, 1205-1211.

11. Chen, L.R.; Wu, S.L.; Shieh, D.T.; Chen, T.R. Sinusoidal-ripple-current charging strategy and optimal charging frequency study for Li-ion batteries. IEEE Trans. Ind. Electron. 2013, 60, 88-97.

12. Chen, L.R.; Chen, J.J.; Ho, C.M.; Wu, S.L.; Shieh, D.T. Improvement of Li-ion battery discharging performance by pulse and sinusoidal current strategies. IEEE Trans. Ind. Electron. 2013, 60, 5620-5628.

13. Hsieh, G.C.; Chen, L.R.; Huang, K.S. Fuzzy-controlled Li-ion battery charge system with active state-of-charge controller. IEEE Trans. Ind. Electron. 2001, 48, 585-593.

14. Chen, L.R.; Hsu, R.C.; Liu, C.S. A design of a grey-predicted Li-ion battery charge system. IEEE Trans. Ind. Electron. 2008, 55, 3692-3701.

15. Petchjatuporn, P.; Sirisuk, P.; Khaehintung, N.; Sunat, K.; Wicheanchote, P.; Kiranon, W. Low cost RISC implementation of intelligent ultrafast charger for $\mathrm{Ni}-\mathrm{Cd}$ battery. Energy Convers. Manag. 2008, 49, 185-192. 
16. Liu, Y.H.; Teng, J.H.; Lin, Y.C. Search for an optimal rapid charging pattern for lithium-ion batteries using ant colony system algorithm. IEEE Trans. Ind. Electron. 2005, 52, 1328-1336.

17. Liu, Y.H.; Luo, Y.F. Search for an optimal rapid-charging pattern for Li-ion batteries using the taguchi approach. IEEE Trans. Ind. Electron. 2010, 57, 3963-3971.

18. Liu, Y.H.; Hsieh, C.H.; Luo, Y.F. Search for an optimal five-step charging pattern for Li-ion batteries using consecutive orthogonal arrays. IEEE Trans. Energy Convers. 2011, 26, 654-661.

19. Chen, B.Y.; Lai, Y.S. New digital-controlled technique for battery charger with constant current and voltage control without current feedback. IEEE Trans. Ind. Electron. 2012, 59, 1545-1553.

20. Lin, F.J.; Huang, M.S.; Yeh, P.Y.; Tsai, H.C.; Kuan, C.H. DSP-based probabilistic fuzzy neural network control for Li-ion battery charger. IEEE Trans. Power Electron. 2012, 27, 3782-3794.

21. Huang, J.W.; Liu, Y.H.; Wang, S.C.; Yang, Z.Z. Fuzzy-Control-Based Five-Step Li-Ion Battery Charger. In Proceedings of the Eighth International Conference on Power Electronics and Drive Systems, Taipei, Taiwan, 2-5 November 2009; pp. 1547-1551.

22. Iranmanesh, H.; Abdollahzade, M.; Miranian, A. Mid-term energy demand forecasting by hybrid neuro-fuzzy models. Energies 2012, 5, 1-21.

23. Chan, Y.; Gu, J. Modeling of turbine cycles using a neuro-fuzzy based approach to predict turbine-generator output for nuclear power plants. Energies 2012, 5, 101-118.

24. Abdelsalam, A.; Cui, S. A fuzzy logic global power management strategy for hybrid electric vehicles based on a permanent magnet electric variable transmission. Energies 2012, 5, 1175-1198.

25. Hsueh, S. A fuzzy utility-based multi-criteria model for evaluating households' energy conservation performance: A taiwanese case study. Energies 2012, 5, 2818-2834.

26. Zhang, Q.; Lai, K.; Niu, D.; Wang, Q.; Zhang, X. A fuzzy group forecasting model based on least squares support vector machine (LS-SVM) for short-term wind power. Energies 2012, 5, 3329-3346.

27. Chan, K.Y.; Khadem, S.; Dillon, T.S.; Palade, V.; Singh, J.; Chang, E. Selection of significant on-road sensor data for short-term traffic flow forecasting using the Taguchi method. IEEE Trans. Ind. Inform. 2012, 8, 255-266.

28. Choi, H.R.; Choe, G.H. A multiobjective parametric optimization for passenger-car steering actuator. IEEE Trans. Ind. Electron. 2011, 57, 900-908.

29. Tarng, Y.S.; Yang, W.H.; Juang, S.C. The use of fuzzy logic in the Taguchi method for the optimisation of the submerged arc welding process. Int. J. Adv. Manuf. Technol. 2000, 16, 688-694.

30. Pandey, A.K.; Dubey, A.K. Taguchi based fuzzy logic optimization of multiple quality characteristics in laser cutting of Duralumin sheet. Opt. Lasers Eng. 2012, 50, 328-335.

(C) 2013 by the authors; licensee MDPI, Basel, Switzerland. This article is an open access article distributed under the terms and conditions of the Creative Commons Attribution license (http://creativecommons.org/licenses/by/3.0/). 